Cinémas

Revue d'études cinématographiques

Journal of Film Studies

Gaudreault, André, Germain Lacasse et Jean-Pierre

Sirois-Trahan. Au pays des ennemis du cinéma... Pour une

nouvelle histoire des débuts du cinéma. Québec : Nuit Blanche

éditeur, 1996, 215 p.

\title{
Pierre Pageau
}

Volume 8, numéro 1-2, automne 1997

Cinéma et mélancolie

URI : https://id.erudit.org/iderudit/024751ar

DOI : https://doi.org/10.7202/024751ar

Aller au sommaire du numéro

Éditeur(s)

Cinémas

ISSN

1181-6945 (imprimé)

1705-6500 (numérique)

Découvrir la revue

Citer ce compte rendu

Pageau, P. (1997). Compte rendu de [Gaudreault, André, Germain Lacasse et Jean-Pierre Sirois-Trahan. Au pays des ennemis du cinéma... Pour une nouvelle histoire des débuts du cinéma. Québec : Nuit Blanche éditeur, 1996, 215 p.]

Cinémas, 8(1-2), 209-213. https://doi.org/10.7202/024751ar d'utilisation que vous pouvez consulter en ligne. 
GAUDREAULT, André, Germain LACASSE et Jean-Pierre SIROIS-TRAHAN. Au pays des ennemis du cinéma... Pour une nouvelle histoire des débuts du cinéma. Québec: Nuit Blanche éditeur, 1996, $215 \mathrm{p}$.

L'intérêt et la connaissance du "cinéma des premiers temps" sont aujourd'hui bien établis. Principalement pour les grandes cinématographies nationales (la France et les États-Unis d'abord, mais aussi l'Italie et la Russie par exemple). Ce qui complète ces études factuelles, et de plus en plus narratologiques, ce sont maintenant des études thématiques (ainsi dans le cadre des colloques de DOMITOR) et des monographies qui visent des secteurs plus délimités. C'est le cas pour cet ouvrage récent sur le cinéma québécois des premiers temps, Au pays des ennemis $d u$ cinéma.

Cependant l'étude du cinéma muet au Québec n'est pas un terrain totalement vierge. Les travaux de Germain Lacasse, un des coauteurs ici, ont déjà fait découvrir plusieurs caractéristiques peu ou pas connues, sur le public, les salles, le bonimenteur, etc. de notre cinématographie. Mais, avec ce livre, on se concentre, d'une façon encore plus pointue, strictement sur le cinéma des origines (1896-1916).

Les différents chapitres (14 en tout) nous mènent de l'arrivée du cinéma au Québec jusqu'aux conditions de l'exploitation en passant par la fabrication et la réception des films. On tentera donc constamment de mettre en relation les films présentés au Québec avec les pratiques sociales d'ordre culturel, économique ou institutionnel. Ceci est d'autant plus nécessaire que la production locale de films demeure assez mince à l'époque. Ce qui ne veut pas dire qu'il n'y a pas eu "activité" cinématographique au Québec. Bien au contraire. 
C'est ainsi que que l'on découvre qu'il y eut très rapidement, au Québec, de nombreux spectateurs pour les projections animées. Un chapitre nous dit que ce fut, au début, des spectateurs "respectables". Par la suite, il y eut des spectateurs plus grossiers qui "s'amusaient à cracher sur les spectateurs du parterre" comme on en voit un équivalent dans Cinéma Paradiso. La salle de cinéma reproduit les classes sociales, mais les réunit néanmoins. Puis, lorsque l'on passera du cinéma forain aux vrais premières salles de cinéma — exemple: le Ouimetoscope de 1906 - il y aura le retour d'un public plus raffiné.

Plusieurs autres faits moins connus sont présentés. Ainsi qu’il y eut plusieurs tentatives de projections de "vues animées coloriées" aussi bien à Québec qu’à Montréal. On fait une bonne recension du travail des journalistes qui s'intéressent à cette nouvelle invention pour découvrir qu'ils sont fort peu critiques. Un chapitre nous rappelle le rôle de la censure dans notre société où le pouvoir clérical jouait un rôle si déterminant. Ce qui nous ramène à la présentation de l'ouvrage où on justifie le titre du livre à partir d'une citation d'un article publié en Russie en 1916 : "Le pays des ennemis du cinéma - Un tel pays existe, non pas dans l'imaginaire, mais bel et bien dans la réalité présente... il s'agit du Québec. » Nous étions les ennemis d'un cinéma amoral et anti-national.

On savait déjà, grâce aux recherches de Germain Lacasse, que la première projection canadienne avait eu lieu le 27 juin 1896 au Palace Theatre du boulevard Saint-Laurent à Montréal. Mais dans cette quête du "premier", dont la critique matérialiste du cinéma nous a déjà dit tant de mal, il fallait aussi chercher le "premier film". On découvre qu’il s'agit d'une actualité des Frères Lumière. L'opérateur Gabriel Veyre, perpétuant la fascination des Français pour les Indiens d'Amérique, filme donc Danse indienne sur la réserve de Kahnawake près de Montréal en 1898. On reconnaît donc bien ici le regard anthropologique conventionnel des opérateurs Lumière; le plus souvent les actualités Lumière n'ont fait que renforcer des stéréotypes quant aux peuples "étrangers" que l'on filmait '.

Cette découverte, parce que c'en est une, du "premier film" est importante. On peut la mettre en parallèle avec la décou- 
verte récente du Cinématographe Lumière \# 16, en très bonne condition et encore prêt pour la projection ${ }^{2}$. Ces deux découvertes témoignent bien de la présence significative des Français en Nouvelle-France, et ce jusqu'en 1916, pour reprendre la périodisation du livre. Par la suite, la présence française se transformera en une présence canadienne (que l'on pourrait aussi traduire par britannique) puis, comme dans bien d'autres pays, en une mainmise états-unienne, reproduisant ainsi l'histoire même de la société québécoise.

Néanmoins, entre 1896 et 1916, la présence française (du Québec et de la France) est très importante: les films Lumière, puis Pathé et Gaumont d'une part, d'autre part des salles de cinéma nationales imposantes bien avant le grand boom des théâtres luxueux états-uniens (1915-1920). En effet, le Ouimetoscope et le Nationoscope, situés en plus l'un près de l'autre, offrent respectivement 1200 et 1100 places et ce, dès 1906 et 1907. Messieurs Ernest Ouimet et Georges Gauvreau se livrent une bataille pour construire le plus beau et grand palace pour attirer le public des notables francophones.

On y apprend par ailleurs que ces deux salles se livrèrent aussi une bataille féroce pour l'installation, dès 1907, de "machines parlantes". Le Nationoscope utilisera le Chronomégaphone Gaumont et le Ouimetoscope le Cinémato-Gramo-théâtre de Georges Mendel. Encore une fois personne, à ma connaissance, n'avait encore été aussi précis dans son constat et son analyse des développements du cinéma sonore dans le cinéma québécois des premiers temps. On y apprend, de plus, que le grand gagnant de cette guerre entre ces deux salles de cinéma fut le Nationoscope, alors que les histoires générales du cinéma québécois ne parlent généralement que de Ouimet et son Ouimetoscope. Fait à noter, le Nationoscope avait des velléités patriotiques et nationales plus affirmées.

Il ne faudrait cependant pas perdre de vue que la majorité des "théâtres" de l'époque appartenait à des anglophones. Donc, durant la période qui nous concerne, on a une présence canadienneanglaise, voire états-unienne, très importante. Cependant, cet ouvrage fait un choix (idéologique?) de présenter davantage un aspect "cinéma québécois francophone», ... culture canadiennefrançaise. On peut parfois s'apercevoir que ce problème de 
définition de la société n'est pas totalement résolu, puisqu'il faut assez souvent se référer à la présence des anglophones pour expliquer la nature du cinéma "québécois". C'est ainsi qu’on nous parle de films réalisés par les États-Uniens sur le territoire québécois: le livre nous présente des photogrammes de films de 1912 de la Biograph, de la Vitagraph et de Lubin. Le plus souvent on utilise le décor de la ville de Québec pour représenter, à un moindre coût, la France. Ce que les États-Uniens font encore de nos jours, comme quoi l'Histoire se répète toujours un peu.

Pourtant, ce livre ne pousse pas son analyse très loin en ce qui concerne cette présence anglophone dans le cinéma québécois d'alors. Alors que l'on sait qu'Edison est déjà présent, avec ses Kinétoscopes dès 1894, et, à partir de 1915, qu'il n'y aura presque plus que du cinéma états-unien sur nos écrans. On ne parle donc pas des propriétaires de salles anglophones et de leurs programmations, des textes trouvés dans les journaux anglophones, sans oublier un grand nombre de projectionnistes ambulantes anglophones. Un numéro récent de la revue Cinémas se référait au "cinéma muet au Québec et au Canada" (vol. 6, $\mathrm{n}^{\circ} 1$ ) et présentait ces interférences sur le territoire du Québec.

Pour mieux comprendre le contexte institutionnel, ce livre nous propose cinq blocs nommés "Documents", qui nous mettent en contact direct avec des écrits de l'époque. Ceci nous rappelle la méthode de Jacques Deslandes dans son Histoire comparée $d u$ cinéma (Casterman, 1969) ou celle, plus vieille, de W. C. Ceram dans son Archéologie du cinéma (Plon 1966). Ces écrits, et de nombreux photogrammes de films invisibles autrement, rendent cet ouvrage encore plus précieux. On ne peut parler d'une critique cinématographique, mais la plupart des textes choisis sont suffisamment étoffés pour que l'on comprenne bien la réalité des premières projections et la perception que l'on a du cinéma, invention technologique fascinante plus que moyen d'expression artistique.

Compte tenu de l'originalité de cette recherche, il ne faut pas se surprendre de ne pas trouver une bibliographie à la fin de l'ouvrage, cependant on y trouve plusieurs pages de référence, en particulier à des journaux de l'époque.

Pierre Pageau 


\section{NOTES}

1 Le film Les Années Lumière, de Jean Chapot (1972) fait une démonstration de cela. C'est un propos biaisé mais stimulant.

2 Dans le cadre de l'exposition "Lumière sur l'istoire de la projection, de la lanterne magique à IMAX ", la Cinémathèque québécoise nous fait voir ce projecteur, le joyau de sa collection. 